\title{
Green chemical approach to fabricate hemp fiber composites for making sustainable hydroponic growth media
}

\author{
Avinash Kumar Both, ${ }^{\dagger}, 1$ Mark Alan Helle, ${ }^{\dagger, 1}$ Guru Madireddy, ${ }^{2}$ and Chin Li Cheung*,1 \\ ${ }^{1}$ Department of Chemistry, University of Nebraska-Lincoln, Lincoln, NE 68588, USA \\ ${ }^{2}$ Department of Mechanical and Materials Engineering, University of Nebraska-Lincoln, \\ Lincoln, NE 68588, USA \\ $\dagger$ These two authors have equal contributions. \\ *Corresponding author; E-mail: ccheung2@unl.edu
}

\section{Electronic Supplementary Information}

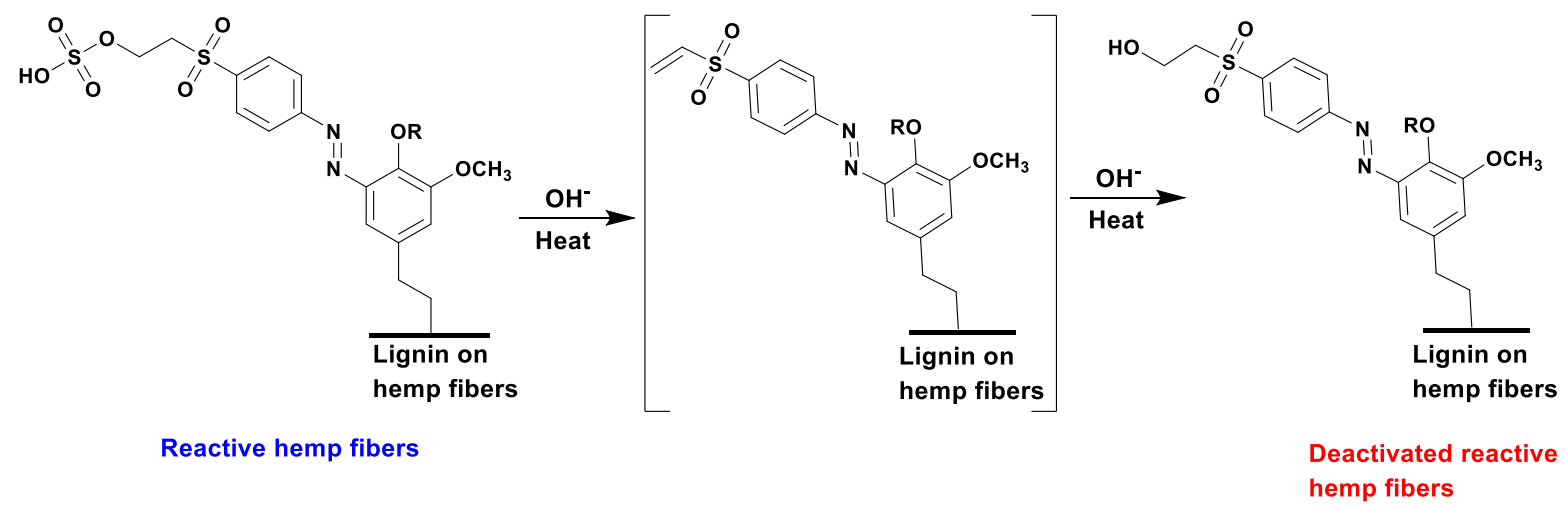

Scheme S1. Reaction scheme showing the deactivation of reactive hemp fibers. The square bracket denotes the vinyl sulfone intermediate in the reaction. 
Table S1. Mechanical properties of hemp fiber composites fabricated with different weight ratios of bifunctional linkers to hemp fibers.

\begin{tabular}{|c|c|c|c|}
\hline $\begin{array}{c}\text { Weight ratio of } \\
\text { bifunctional linker: hemp fiber }\end{array}$ & Hemp fiber composite & Bulk density $\left(\mathrm{g} / \mathrm{cm}^{3}\right)$ & Compressive strength (MPa) \\
\hline $3: 5$ & & 0.52 & $1.81 \pm 0.02$ \\
\hline $3: 10$ & & 0.31 & $1.64 \pm 0.02$ \\
\hline $3: 20$ & & 0.30 & $1.36 \pm 0.03$ \\
\hline 3:40 & & 0.26 & $0.91 \pm 0.04$ \\
\hline
\end{tabular}

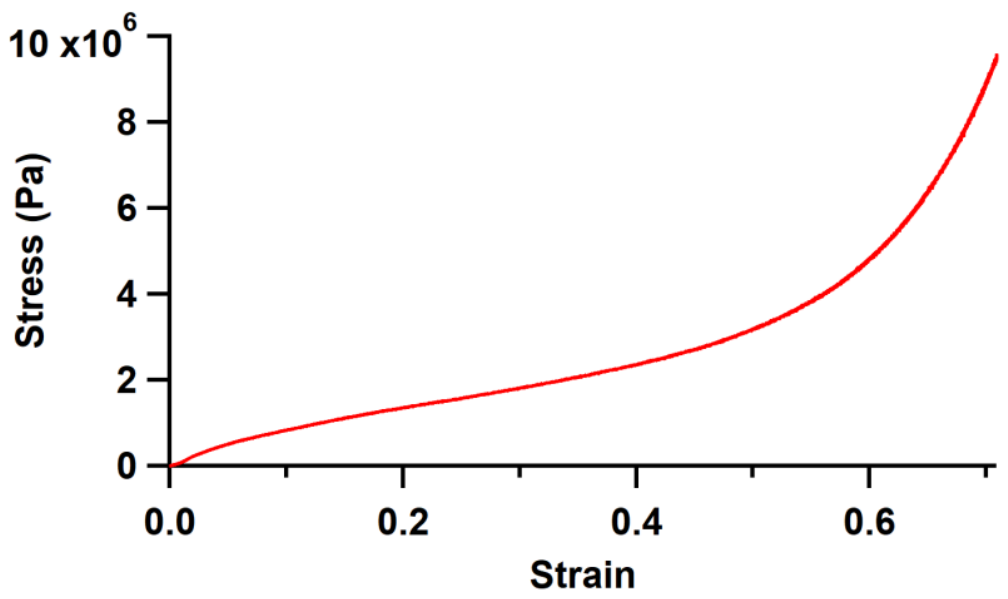

Figure S1 Plot depicting the stress-strain curve of the mechanical compression study of a hemp fiber composite fabricated with a 3:10 weight ratio of bifunctional linkers to hemp fibers. 
Video SV1. Compressive mechanical evaluations of the control samples at the compression rate of $2 \mathrm{~mm}$ per minute. The video speed has been increased by 30x times. (File: as1c00118_si_004.mp4)

Video SV2. Compressive mechanical evaluations of as-produced hemp fiber composites fabricated with a 3:10 weight ratio of bifunctional linkers to hemp fibers at the compression rate of $2 \mathrm{~mm}$ per minute. The video speed has been increased by 30x times. (File: as1c00118_si_005.mp4)



Day-6

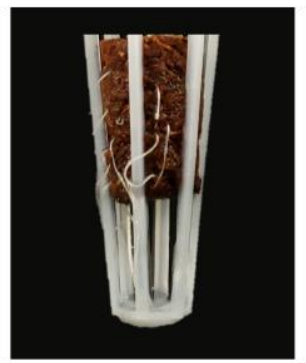

Day-8

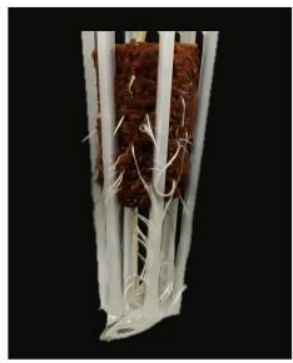

Day-10

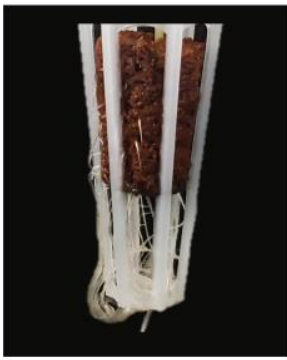

Day-12

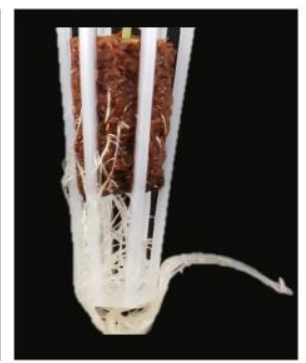

Day-14

Figure S2. Photos showing the root growth of a Daikon radish plant using a hemp fiber composite fabricated with a 3:10 weight ratio of bifunctional linkers to hemp fibers as a hydroponic growth medium.

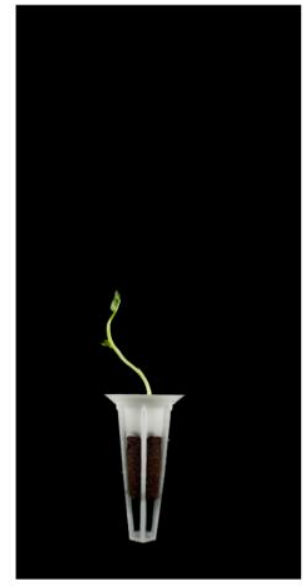

Day-6

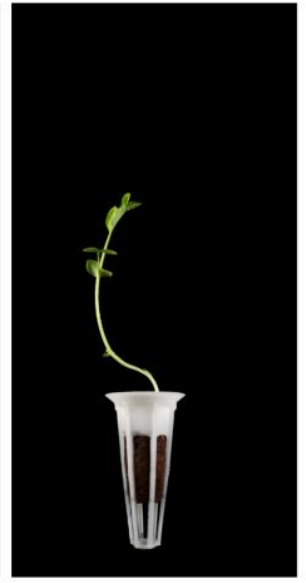

Day-8

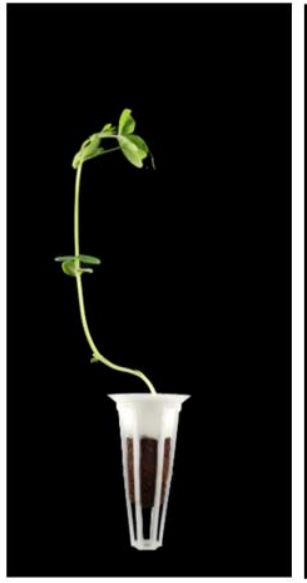

Day-10

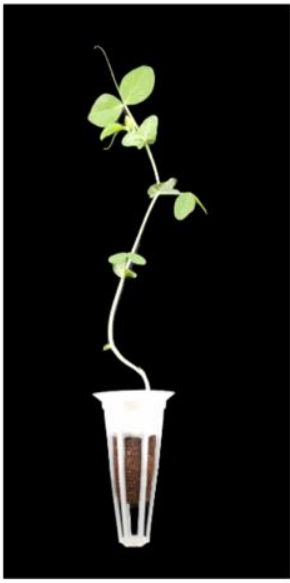

Day-12



Day-14

Plant Growth Progress in 14 Days

Figure S3. Photos showing the hydroponic growth of a green pea (Pisum sativum) plant using a hemp fiber composite growth medium from Day-6 to Day-14. 
Table S2. Table depicting growth parameters employed to study the hydroponic growth of a green pea plant with the hemp fiber composite fabricated with a 3:10 weight ratio of bifunctional linkers to hemp fibers as the growth medium

\begin{tabular}{|c|c|c|}
\hline Growth day & Number of leaves & Plant height $(\mathbf{c m})$ \\
\hline Day-6 & 3 & 7.5 \\
\hline Day- 8 & 10 & 11.4 \\
\hline Day-10 & 13 & 15.8 \\
\hline Day-12 & 16 & 19.0 \\
\hline Day-14 & 21 & 20.1 \\
\hline
\end{tabular}

